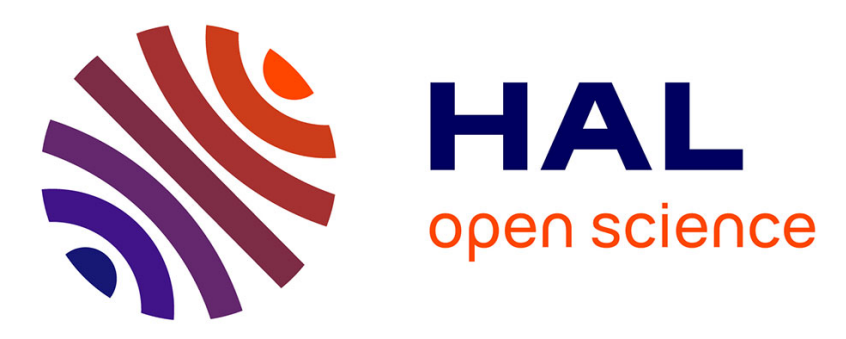

\title{
Fractionation of grape anthocyanin classes using multilayer coil countercurrent chromatography with step gradient elution
}

Stéphane Vidal, Yoji Hayasaka, Emmanuelle Meudec, Veronique Cheynier, George Skouroumounis

\section{To cite this version:}

Stéphane Vidal, Yoji Hayasaka, Emmanuelle Meudec, Veronique Cheynier, George Skouroumounis. Fractionation of grape anthocyanin classes using multilayer coil countercurrent chromatography with step gradient elution. Journal of Agricultural and Food Chemistry, 2004, 52 (4), pp.713-719. 10.1021/jf034906a . hal-01981161

\section{HAL Id: hal-01981161 https://hal.science/hal-01981161}

Submitted on 14 Jan 2019

HAL is a multi-disciplinary open access archive for the deposit and dissemination of scientific research documents, whether they are published or not. The documents may come from teaching and research institutions in France or abroad, or from public or private research centers.
L'archive ouverte pluridisciplinaire HAL, est destinée au dépôt et à la diffusion de documents scientifiques de niveau recherche, publiés ou non, émanant des établissements d'enseignement et de recherche français ou étrangers, des laboratoires publics ou privés. 


\title{
Fractionation of Grape Anthocyanin Classes Using Multilayer Coil Countercurrent Chromatography with Step Gradient Elution
}

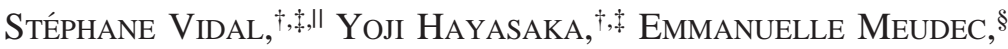

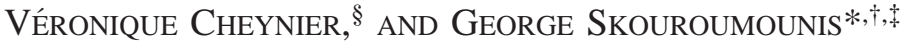

\begin{abstract}
The Australian Wine Research Institute, PO Box 197, Glen Osmond SA 5064, Australia, Cooperative Research Centre for Viticulture, PO Box 154, Glen Osmond SA 5064, Australia, and Unité Mixte de Recherche Sciences pour l'Oenologie, INRA, 2, place Viala, 34060 Montpellier cédex, France
\end{abstract}

\begin{abstract}
Grape anthocyanins from rose wine pomace and grape skins were fractionated by multilayer coil countercurrent chromatography (MLCCC). Tert-butyl methyl ether/n-butanol/acetonitrile/water acidified with trifluoroacetic acid $(2 / 2 / x / 5)$ was chosen as the solvent system because of its demonstrated efficiency in separating anthocyanins. A method combining MLCCC and step gradient elution has been developed. This method enabled the fractionation of anthocyanins as series of glucosides, and the corresponding acetylated, coumaroylated, and caffeoylated derivatives. The different anthocyanins were identified on the basis of their UV spectra, their elution time on reversed-phase HPLC as well as by mass spectrometry. A polar-colored fraction free of anthocyanin monomers and corresponding to the material remaining in the stationary phase after the completion of the gradient was recovered.
\end{abstract}

KEYWORDS: Grape; anthocyanins; liquid-liquid separation; multilayer coil countercurrent chromatography; step gradient elution

\section{INTRODUCTION}

Anthocyanins from Vitis vinifera grapes consist of malvidin, peonidin, petunidin, cyanidin, and delphinidin 3-D-glucosides and the corresponding acetylated, caffeoylated, and $p$-coumaroylated esters (Figure 1). Anthocyanins are responsible for the color of young red wines, and their effectiveness as food colorants is $\mathrm{pH}$-dependent. Apart from their color properties, their sensory properties are still not well understood. Although recent studies have suggested that they could contribute to fullness $(1)$ and astringency $(1,2)$, this remains to be confirmed. In aqueous media, anthocyanins exist in different $\mathrm{pH}$-dependent forms that are diversely chemically reactive and as such undergo different types of reactions occurring during wine aging, resulting in the formation of newly derived pigments, as it has been reviewed (3). Furthermore, reactions with other grapederived or exogenous molecules increase the degree of complexity in studying their sensorial characteristics. These miscellaneous reactions involving anthocyanins have been hypothesised to decrease wine astringency (3). In addition to their sensory characteristics, anthocyanins also display antioxidant properties (4).

Further development of techniques to fractionate and isolate anthocyanins is necessary to develop a better understanding of

* To whom correspondence should be addressed. Fax: 61883036601 . E-mail: George.Skouroumounis@awri.com.au.

The Australian Wine Research Institute.

$\doteqdot$ Cooperative Research Centre for Viticulture.

$\S$ Unité Mixte de Recherche Sciences pour l'Oenologie.

" Present address: Inter Rhône, 2260 route du Grès, 84100 Orange, France. the complexity of their intrinsic and reactivity-related properties. To illustrate the latter, the reaction of isolated anthocyanins with specific wine components under defined model conditions could represent a potent way to produce specific wine pigments $(5-7)$. This could avoid the need to fractionate the different kinds of pigments formed during wine aging, which remains a difficult task due to the complex nature of the matrix (3).

As an alternative to the classical gel chromatographic techniques, countercurrent chromatography has been recently examined and has proven to be effective in the preparative fractionation of anthocyanins (8). The principle of this technique is first the retention of a liquid stationary phase on a Teflon tube by the use of a high centrifugal force. This is followed by the use of an immiscible mobile phase, which is then pumped through the stationary phase, ensuring complete contact with the stationary phase and thus allowing the partitioning of the analytes between the two phases. A hydrodynamic equilibrium is established between the two immiscible solvent phases as they distribute unilaterally in a rotating coil, one phase entirely occupying the head side and the other phase the tail side of the coil, with the definition of head and tail side of the coil being dependent on how the coil is wound in the bobbin or holder and the direction of the rotating axis. Due to the planetary motion of the coils, a sample injected within the mobile phase will experience approximately 50000 mixing and settling steps an hour (9). Generally, as the two immiscible layers have an auxiliary solvent that is miscible in both liquid phases, the auxiliary solvent aids in the partitioning of the analytes between the phases. Therefore, partitioning occurs due to the intrinsic solubilities of the analytes within the two phases, as the mobile phase 


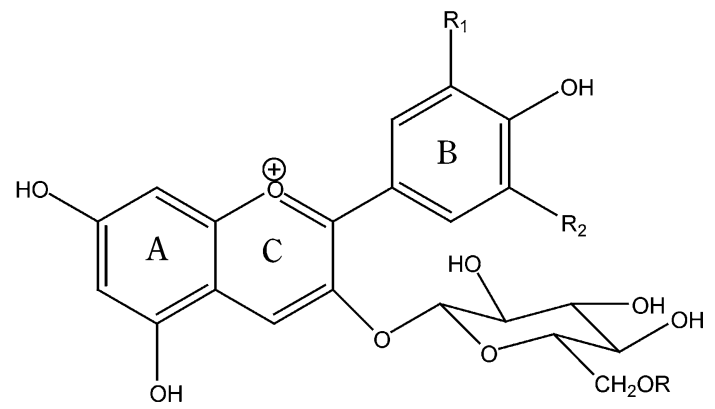

A<smiles>[R]C(=O)/C=C/c1ccc(O)c([R3])c1</smiles>

\begin{tabular}{|c|c|c|c|c|c|c|}
\hline $\mathrm{A}$ & R1 & $\mathbf{R 2} \mathrm{R}$ & $\mathrm{z}=\mathrm{H}$, glucoside & $\mathrm{R}=$ acetyl-glc ${ }^{\mathrm{a}}$ & $R=$ caffeoyl-glc & $\mathrm{R}=p$-coumaroyl-glc \\
\hline Malvidin & $\mathrm{OCH}_{3}$ & $\mathrm{OCH}_{3}$ & $q$ & 1 & g & $\mathrm{a}$ \\
\hline Peonidin & $\mathrm{OCH}_{3}$ & $\mathrm{H}$ & $\mathrm{r}$ & $\mathrm{m}$ & h & b \\
\hline Petunidin & $\mathrm{OCH}_{3}$ & $\mathrm{OH}$ & $\mathrm{s}$ & $\mathrm{n}$ & I & $\mathrm{c}$ \\
\hline Cyanidin & $\mathrm{OH}$ & $\mathrm{H}$ & $\mathrm{t}$ & o & $\mathrm{j}$ & d \\
\hline Delphinidin & $\mathrm{OH}$ & $\mathrm{OH}$ & $\mathrm{u}$ & $\mathrm{p}$ & $\mathrm{k}$ & $\mathrm{e}$ \\
\hline
\end{tabular}

a Glucoside portion without the $H$ in $R$ substructure $A$, abbreviated as glc

Figure 1. Structures of the different native anthocyanins. For each class, anthocyanins have been labeled in an alphabetical order following their order of elution by reversed-phase HPLC.

is passed through the coils. Using this all liquid-liquid chromatographic technique, most interactions between anthocyanins and solid support are avoided, and therefore there is essentially no sample loss (9). The flexible use of biphasic solvent systems represents another advantage of this technique. The use of the solvent system of tert-butyl methyl ether/n-butanol/acetonitrile/ water acidified with trifluoroacetic acid $(2 / 2 / 1 / 5)$ was found to be suitable for anthocyanin separation $(8)$.

The aim of the present study was to develop a technique to separate the different classes of anthocyanins (glucosides, acetyl glucosides, caffeoyl glucosides, and coumaroyl glucosides) on a gram scale and in a relatively short time. These materials will be essentially used for sensory evaluation but will also serve as reference compounds and be useful for model studies and structural elucidation. Furthermore, an insight gained by changing the polarities of a single solvent in a multisolvent system can affect separation of a complex matrix.

\section{MATERIALS AND METHODS}

Anthocyanin Sources. Marc Extract. Marc extract was obtained from Société Française de Distillerie (Vallon-Pont-d'Arc, France). This material results from an industrial extraction of polyphenolic compounds from a rosé wine marc followed by concentration of the extract on resins.

Grape Skin Material. Skins (600 g) were manually recovered from frozen grape berries of Vitis vinifera $\mathrm{cv}$. Shiraz harvested at commercial maturity at the INRA experimental station of Pech-Rouge. Skins were frozen in liquid nitrogen and ground using a Dangoumau blender (Prolabo, France). The resulting powder was extracted at $4{ }^{\circ} \mathrm{C}$ overnight with $7 \mathrm{~L}$ of ethanol/water (75/25) containing $2 \%$ of acetic acid. Ethanol/ water $(75: 25 ; \mathrm{v} / \mathrm{v})$ was used to favor the extraction of anthocyanins over that of proanthocyanidins that are known to require solvents such as methanol or acetone/water $(60: 40 ; \mathrm{v} / \mathrm{v})$ to be quantitatively extracted (10). The extract was sieved on nylon mesh (Scrynel, $500 \mu \mathrm{m}$, Poly Labo, Strasbourg, France) and filtered through GF/C glass microfiber filter (Whatman, Maidstone, England).

The extract was concentrated under vacuum to $2 \mathrm{~L}$ at $35^{\circ} \mathrm{C}$. This solution contained $6 \mathrm{~g}$ of malvidin 3-glucoside equivs (as determined by HPLC-DAD) in total. This extract was loaded onto $11 \mathrm{~L}$ of Toyopearl TSK HW-50(F) gel (Tosoh Corp., Tokyo, Japan) packed in a semipreparative scale column $(50-\times 25-\mathrm{cm})$ equilibrated in water. The working flow rate was set at $100 \mathrm{~mL} / \mathrm{min}$ and monitored by a Dynamax SD-1 pump (RAININ). The chromatographic conditions were adapted from a recently published study dealing with the fractionation of anthocyanin-related materials (11). The column was sequentially washed with water (3 bed volumes), ethanol/water/TFA (20:80:0.05, $\mathrm{v} / \mathrm{v} / \mathrm{v})$ ( 2 bed volumes) to recover the main part of anthocyanin glucosides and with ethanol/water/TFA (80:20:0.05, v/v/v) to recover the remaining anthocyanins. The latter fraction, referred to as skin extract, was lyophilised, and the resulting red powder was stored at room temperature under nitrogen and used for fractionation of anthocyanins.

MLCCC System. The MLCCC system was a Quattro (Analytical and Enviromental Consultancy Services (AECS), Bridgend, S. Wales, United Kingdom) equipped with four coils on two holders (bobbins of 190-mm diameter). Each bobbin is composed of two coils (each with a 1.6-mm internal diameter Teflon tubing (ID)) with volume capacity of $100-\mathrm{mL}$ and $250-\mathrm{mL}$, respectively. Each coil is equipped with two flying leads $(0.5 \mathrm{~mm}-\mathrm{ID})$, allowing coils to be connected in series or operated separately. By interconnecting different combinations of coils, it was possible to use different volumes of stationary phase from 100 $\mathrm{mL}$ to $700 \mathrm{~mL}$. The temperature of the cabinet containing the different coils was controlled by a thermostated cooling system (Ratek circulated cooling device, Adelab, Adelaide, Australia). The whole system was 
equipped with a backpressure regulator (250 psi), Upchurch Scientific, New Zealand) set upstream of the bobbins.

Optimum conditions for the separation of $5 \mathrm{~g}$ of skin material was performed by multiple runs of $250-\mathrm{mg}$ injections (in 2-mL sample loop) onto a 200-mL coil (two 100-mL coils connected in series, also known as the outer coils in each bobbin). The other two coils (two $250-\mathrm{mL}$ coils, also known as the inner coils in each bobbin) were not used, but filled with water as a counter balance for each bobbin. Revolution speed was set at $800 \mathrm{rpm}$ (revolution per minute), temperature kept at $25^{\circ} \mathrm{C}$, and the flow rate was delivered at $2 \mathrm{~mL} / \mathrm{min}$ by a Waters $600 \mathrm{E}$ pump, the elution being monitored by a UPC-900 detector (Amersham Pharmacia Biotech, Sweden) set at $546 \mathrm{~nm}$. The samples were dissolved in a (50:50; v/v) mixture of the upper and lower phases of the solvents prior to injection through a Rheodyne injection loop. Coil volumes varied $(100-700 \mathrm{~mL})$ depending on the sample size during method development. The amount of sample loaded on the system ranged from $100 \mathrm{mg}$ to $2 \mathrm{~g}$ with Rheodyne injection loop minimum/maximum size of $2 / 5 \mathrm{~mL}$.

Analytical Methods. HPLC-DAD/ESI-MS. HPLC analyses were performed using a Waters Millenium HPLC-DAD system (Milford, MA) as described previously (12).

ESI-MS/MS analyses were performed with a ThermoFinnigan LCQ Advantage (San Jose, California) mass spectrometer equipped with an electrospray source and an ion trap mass analyzer controlled by the LCQ navigator software. The spectrometer was operated in the positive ion mode (source voltage, $4.5 \mathrm{kV}$, capillary voltage, $23.5 \mathrm{~V}$; capillary temperature, $250^{\circ} \mathrm{C}$. Collision energy for fragmentation used for $\mathrm{MS}^{2}$ experiments were set at $35 \%$.

Thiolysis. The thiolytic reagent was a $5 \%$ solution of phenylmethanethiol in methanol containing $0.2 \mathrm{M} \mathrm{HCl}$. Each fraction $(2 \mathrm{mg}$ dissolved in $200 \mu \mathrm{L}$ of methanol) was dissolved in the methanol/ thiolytic reagent $(1: 1 ; \mathrm{v} / \mathrm{v})$ and heated for $2 \mathrm{~min}$ at $90{ }^{\circ} \mathrm{C}$. The released units were analyzed by HPLC under the conditions previously described (13). Quantification of each terminal and extension unit was based on peak areas at $280 \mathrm{~nm}$, and calibration was undertaken with purified external standards (13).

\section{RESULTS AND DISCUSSION}

Development and Optimization of MLCCC Operating Conditions. The MLCCC method using step gradient elution was developed using a solution of rosé wine pomace on one coil of $100 \mathrm{~mL}$, with the other $100 \mathrm{~mL}$ coil being used as a counter-balance and both coils loaded with the stationary phase. This industrially enriched anthocyanin preparation was available in large amounts. It contained $90 \mathrm{mg}$ of anthocyanins (expressed as malvidin 3-glucoside equivs) per gram of powder (calculated by high performance liquid chromatography as described above).

Finding an appropriate solvent system for sample partition can prove difficult. For this reason, the multisolvent system composed of tert-butyl methyl ether/n-butanol/acetonitrile/water acidified with $0.1 \%$ (v/v) trifluoroacetic acid (TFA) (2/2/1/5), which had already proven to be efficient (9) regarding anthocyanin fractionation, was retained. This system has been previously used for the isolation of anthocyanins from different sources such as red cabbage, black currant and black chokeberry (8). In another study, the separation of the different classes of anthocyanins present in wine had been performed by using different solvent systems of different polarities (i.e., tert-butyl methyl ether/ $n$-butanol/acetonitrile/water acidified with $0.1 \%$ (v/v) TFA (2/2/1/5) for the glucosides, ethyl acetate/water $(1 / 1)$ plus $0.1 \%$ (v/v) TFA for the coumaroyl- and caffeoylglucosides and ethyl acetate/n-butanol/water (4/1/5) plus $0.1 \%$ (v/v) TFA for the acetyl-glucosides) (9).

Gradient elution combined with centrifugal partition chromatography (CPC) has already been used (14) to purify anthocyanins from Champagne byproducts using the ethyl acetate $/ n$-butanol/water $(0.2 \%$ TFA $)$ ternary solvent. In this
Table 1. Step Gradient Elution

\begin{tabular}{rcc}
\hline time & & \\
$(\mathrm{min})$ & \% solvent A & \% solvent B \\
\hline 0 & 100 & 0 \\
10 & 54 & 46 \\
100 & 30 & 70 \\
160 & 30 & 70 \\
\hline
\end{tabular}

work, the authors varied each solvent system of the gradient using the appropriate tie-lines and ensuring the lower aqueous phase was roughly identical for all the biphasic mixtures and immiscible with the corresponding organic phases. The group of acylated anthocyanins was separated from the glucosides that were themselves chromatographed in three fractions, namely peonidin 3-glucoside, malvidin 3-glucoside, and a mixture of cyanidin 3-glucoside with delphinidin 3-glucoside and petunidin 3-glucoside. In the current study, it was decided to vary the polarity of the biphasic solvent system by changing the ratio of one of the auxiliary solvents. This was chosen to make the protocol easy to carry out, as no reference to a complex tableto-determine ratio of each solvent is needed. Varying the acetonitrile content of the biphasic system while keeping parts of the other solvents constant was chosen to perform the step gradient. We determined the workable range of acetonitrile content using Ito's method by determining at which acetonitrile content the emulsion occurring between the two phases after shaking (in the presence or absence of the sample to be partitioned) remained for less than $30 \mathrm{~s}$ (15). The system of tert-butyl methyl ether/n-butanol/acetonitrile/water acidified with $0.02 \%(\mathrm{v} / \mathrm{v})$ TFA $(2 / 2 / x / 5)$, with $x$ varying between 0.1 and 2.5 was compatible with MLCCC use.

In many of the systems mentioned above (using MLCCC), the organic phase was used as the stationary phase, as it would be easier to freeze-dry the aqueous eluting mobile phase once separation had occurred. However, better phase retention was obtained when the aqueous phase was used as the stationary phase. Consequently, a "tail to head" elution mode was adopted. As the phase retention is temperature dependent, different temperature conditions were studied $\left(14-35^{\circ} \mathrm{C}\right)$, as well as different rotational speeds $(600-800 \mathrm{rpm})$. The best conditions to achieve consistent high stationary phase retention was at a temperature of $25^{\circ} \mathrm{C}$, with a rotational speed of $800 \mathrm{rpm}$. Under these conditions, the stationary phase retention was in the range of $75-85 \%$. During the developmental stage, the separation of anthocyanins was monitored by HPLC, and the various fractions were confirmed by use of LC-ESIMS. The optimized MLCCC process was determined as follows: The two 100-mL coils were connected in series and filled with the lower phase of the system of tert-butyl methyl ether/n-butanol/acetonitrile/water acidified with $0.02 \%(\mathrm{v} / \mathrm{v})$ TFA $(2 / 2 / 0.1 / 5 ; \mathrm{v} / \mathrm{v} / \mathrm{v} / \mathrm{v})$. After equilibrating the system by pumping the corresponding upper phase through, the elution was performed following a binary stepwise gradient of solvent A (upper phase of the sytem tert-butyl methyl ether/n-butanol/acetonitrile/water acidified with $0.02 \%(\mathrm{v} / \mathrm{v})$ TFA (2/2/0.1/5; v/v/v/v)) and solvent B (upper phase of the system tert-butyl methyl ether/ $n$-butanol/acetonitrile/water acidified with $0.02 \%(\mathrm{v} / \mathrm{v})$ TFA $(2 / 2 / 2.5 / 5 ; \mathrm{v} / \mathrm{v} / \mathrm{v} / \mathrm{v}))$. The optimized conditions of the step gradient are shown in Table $\mathbf{1}$.

Sample Preparation. The sample preparation holds importance, as the mode of liquid/liquid chromatography is dependent on a number of factors. One important factor is the sample matrix, which can affect the polarity of the whole system and therefore the chromatography process. During developmental stages of the method, we noted that the use of a commercially 


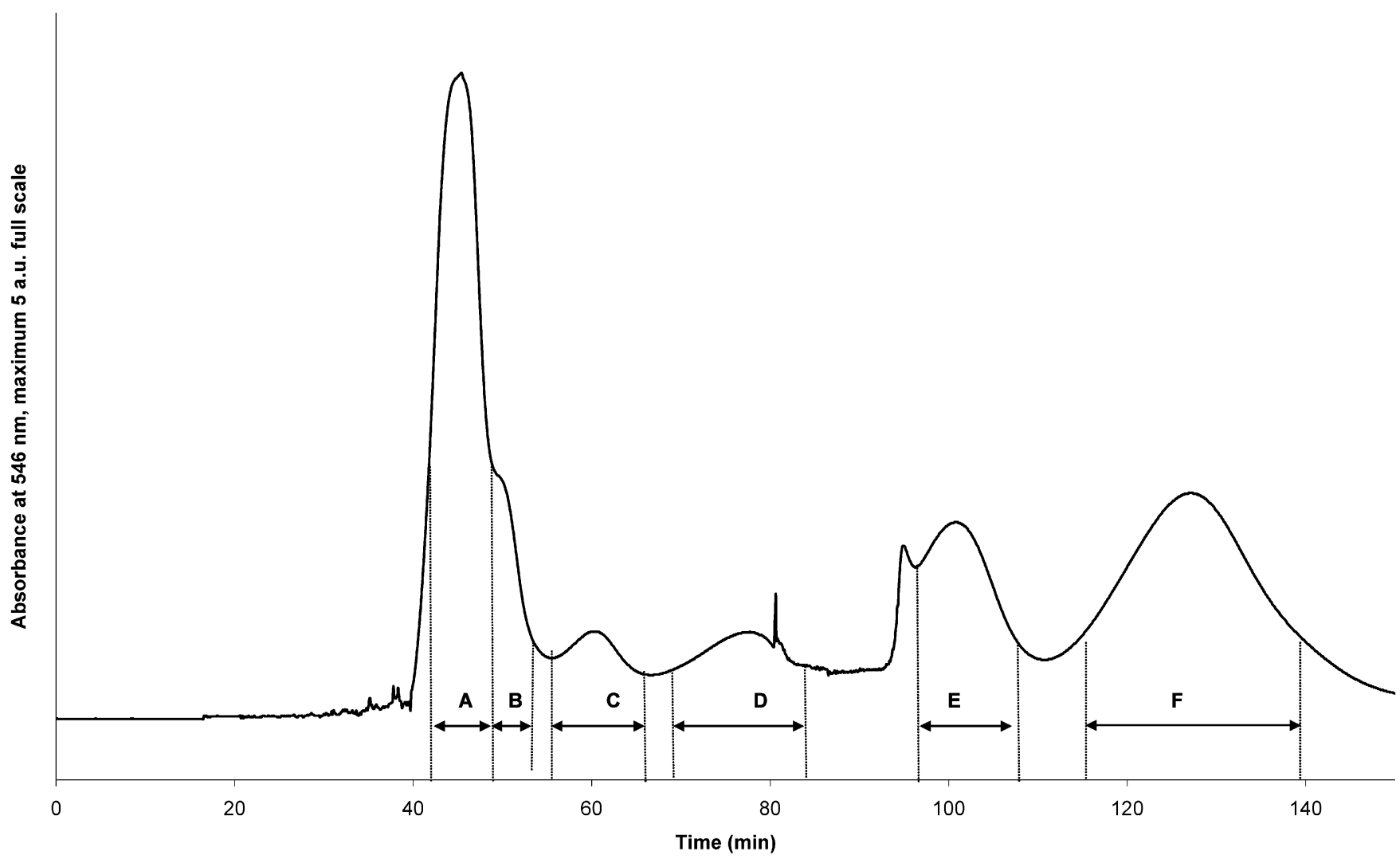

Figure 2. Profile of elution monitored at $546 \mathrm{~nm}$. The different fractions were collected as indicated by the dotted lines.

available enriched liquid anthocyanin extract dramatically influenced the retention times, but the order of peak elution was still the same (data not shown). We thus decided to use powdered samples (a grape skin extract and an industrially produced marc extract) to obtain reproducible chromatography. The industrially enriched anthocyanin preparation was obtained from marc by a process of separation on resins allowing the removal of polar compounds such as proteins and polysaccharides and by our request was not supplemented with any stabilizers. In the case of the grape skin extract, the use of $75 \%$ ethanol for the extraction step allowed the elimination of most grape polysaccharides and proteins by precipitation. As a consequence, the two extracts used in our study gave us similar elution profiles.

Isocratic Elution. An isocratic elution using 0.1 parts of acetonitrile in the solvent mixture was time-consuming and not appropriate for preparation of large quantities of each class of anthocyanins. Isocratic elution has been used in the past, but step gradient elution does offer much more flexibility to the conditions at hand.

Step Gradient Elution. A knowledge of the stationary phase selection and retention is important in working out the step gradient conditions to use for the mobile phase. The required volumes of mobile phase for each step of the gradient can then be calculated to allow $2-3$ mobile phase volumes. For example, if $70 \%$ stationary phase retention is observed in a $100-\mathrm{mL}$ coil, then $30 \mathrm{~mL}$ of mobile phase correponds to one column volume in the system. To allow 2 volumes of mobile phase, $60 \mathrm{~mL}$ of each step are required and would correspond to $30 \mathrm{~min}$ in the case of $2 \mathrm{~mL} / \mathrm{min}$ flow rate. The lower phase of solvent $\mathrm{A}$, which is more polar than the lower phase of solvent $\mathrm{B}$, was selected as the stationary phase. As each step gradient was planned to account for two mobile phase volumes, it was important to determine how many steps were performed in a step gradient. Initially, a five step gradient was planned (acetonitrile $=0.1$,
$0.3,0.6,0.9,1.2$ parts), but the separation took over $3 \mathrm{~h}$. The number of gradient steps was modified to shorten the chromatographic process while keeping the same level of resolution. It was then considered to use fewer gradient steps and to increase the polarity of the mobile phase earlier on in the step gradient (acetonitrile $=0.1,1.2$, and 1.8 parts) to decrease the elution times of the different classes of anthocyanins. In the first step of the gradient elution, less than one mobile phase volume was considered, as the initial acetonitrile concentration had no drastic affect on the time of elution of the early-eluted compounds. The second and third steps of the gradient elution were optimized to 3 mobile phase volumes. The conditions retained are given in Table $\mathbf{1}$.

Separation Profile Using Step Gradient. The step gradient conditions developed with the rosé wine pomace were used for the separation of the different anthocyanin classes from the skin extract. This skin polyphenolic material was chosen because of its high anthocyanin content. Skin material $(5 \mathrm{~g})$ was fractionated in 20 runs of $0.25 \mathrm{~g}$ per run, using the chosen method described in the previous paragraph

The UV (546 nm) absorbance profile (Figure 2) was dominated by fraction A, eluting between 40 and $47 \mathrm{~min}$ followed by peak B as a shoulder. Four other fractions (C, D, $\mathrm{E}$, and F) were eluted later in the gradient. The fractionation observed was reproducible from one injection to another, and the fractions collected in successive elutions as indicated by the dotted lines in Figure $\mathbf{2}$ were pooled together. The preparative fractionation yielded $1180 \mathrm{mg}$ of fraction $\mathrm{A}, 183$ $\mathrm{mg}$ of fraction $\mathrm{B}, 63 \mathrm{mg}$ of fraction $\mathrm{C}, 83 \mathrm{mg}$ of fraction $\mathrm{D}$, $100 \mathrm{mg}$ of fraction E, and $190 \mathrm{mg}$ of fraction F. Along with these fractions, $175 \mathrm{mg}$ of the material remaining in the stationary phase was also collected. From the $5 \mathrm{~g}$ chromatographed, around $2.2 \mathrm{~g}$ was collected, corresponding to a recovery yield of $45 \%$. This somewhat low yield may be attributable to 
the mode of collection used to avoid overlapping fractions. The rest was spread out in the non collected fractions.

Characterization of the Different Fractions. Analysis of the fractions was performed by LC-DAD-ESI-MS. The relative proportions of the different anthocyanin classes in each fraction were evaluated from the $520 \mathrm{~nm}$ traces. The presence of proanthocyanidin-based polyphenolic (proanthocyanidins and derived pigments) structures was investigated in each fraction by acid-catalyzed depolymerization in the presence of a thiolytic agent (thiolysis) (16). This technique discriminates between terminal units that are released as flavan-3-ol monomers and extension units released as thiol derivatives resulting from the trapping of the cation generated through the breakdown of the interflavan linkage by the thiolytic agent. None of the fractions collected in this study yielded any of these flavanol units.

Fraction A was mainly composed of the five anthocyanin coumarates (Figure 3, compounds a-e), with malvidin 3-coumaroylglucoside accounting for $60 \%$ of the coumaroylated forms. Malvidin and peonidin 3-caffeoylglucoside were also present $(\mathrm{g}$ and $\mathrm{h})$ and accounted, when taken together, for $8 \%$ of the $520 \mathrm{~nm}$ trace recorded for this fraction. Other phenolic compounds present in this fraction that accounted for $13 \%$ of the $280 \mathrm{~nm}$ trace were also detected (data not shown). They were essentially flavonols, identified on the basis of their UVvis spectra, retention time, and mass spectral $\mathrm{m} / \mathrm{z}$ values for M $+\mathrm{H}^{+}$. They included quercetin 3-glucoside $(\mathrm{m} / \mathrm{z}, 465)$, quercetin 3-glucuronide $(\mathrm{m} / \mathrm{z} 479)$, myricetin 3-glucuronide $(\mathrm{m} / \mathrm{z} 495)$, and isorhamnetin 3-glucoside $(\mathrm{m} / \mathrm{z} 479)$. The $520 \mathrm{~nm}$ trace of fraction B was dominated by anthocyanidin 3-caffeoylglucosides (55\%; compounds $\mathrm{g}-\mathrm{k}$ ), although this fraction still contained a large amount of coumarates (33\%, compounds a-d) because of the poor resolution between the two fractions. Anthocyanidin 3 -acetylglucosides explained the remaining $12 \%$. All the anthocyanins accounted for around $90 \%$ of the $280 \mathrm{~nm}$ trace, flavonols being predominant among the remaining species.

Anthocyanidin 3-acetylglucosides were fractionated in two peaks (fractions $\mathrm{C}$ and D), as were the glucosides (peaks E and F). In both cases, the separation in each class was based on the degree of substitution on the B ring of the anthocyanins (Figure 1). Cyanidin and peonidin, which are marked by the lowest degree of hydroxylation/methoxylation of the B ring, were eluted before petunidin, delphinidin, and malvidin either in their glucoside or acetyl glucoside forms. In this study, where the molecules are eluted with an increasing polarity, order of elution did not inversely mirror those observed by reversed-phase HPLC. This sort of difference between all-liquid and solidsupport chromatography was reported in previous studies $(8$, 14). Whether this difference is based only on the respective polarity of the anthocyanins and/or the special interactions these molecules can establish with the solid phase in classical HPLC columns or with solvent molecules in all-liquid chromatography is difficult to ascertain.

The 520-nm trace of fraction $\mathrm{C}$ was dominated by peonidin 3-acetylglucoside (m, 60\%). Cyanidin 3-acetylglucoside (o) and malvidin 3-acetylglucoside (1) represented only minor components of this fraction. Peonidin- (r) and malvidin 3-glucoside (q) $(8 \%)$ and their corresponding coumaroylated derivatives (6\%) were also detected, as well as malvidin 3-caffeoylglucoside (approx $1 \%)$. Other species $(\mathrm{m} / \mathrm{z}, 623,653,627$, and 657) were detected but could not be easily identified. LC-MS/MS experiments were carried out to further investigate their structure. This technique revealed that molecular ions at $\mathrm{m} / \mathrm{z}, 623$ and $\mathrm{m} / \mathrm{z}, 653$ on one hand and $\mathrm{m} / \mathrm{z} 627$ and $\mathrm{m} / \mathrm{z} 657$ on the other hand are structurally related, as they showed a common fragmentation pattern with a mass difference of $30 \mathrm{amu}$. These compounds exhibited a spectrum characterized with a $\lambda_{\max }$ around $540 \mathrm{~nm}$, suggesting that they consist of derived anthocyanins (17). Their fragmentation did not produce ions that could be identified as aglycones corresponding to any of the five different native anthocyanidins. Unlike the compounds giving $\mathrm{m} / \mathrm{z}, 627$ and 657 ions, no loss of 162 amu could be observed in the fragmentation pathway of compounds, giving $\mathrm{m} / \mathrm{z} 623$ and $\mathrm{m} / \mathrm{z} 653$ ions, suggesting that these types of pigments were not glycosylated.

The composition of fraction D is very similar to that of fraction $\mathrm{C}$, with the exception of a rather low concentration of peonidin 3-acetylglucoside $(\mathrm{m})$ and the predominance of the three acetates of malvidin (1), petunidin (n), and delphinidin (p) 3-glucosides that accounted for $75 \%$ of the compounds contributing to the 520-nm trace. The five glucosides are also present in a smaller amount (17\%), the main one being peonidin 3-glucoside that accounts for less than the delphinidin 3-acetyl glucoside on the basis of their respective area. The proportion of glucosides in this fraction was increased after sample evaporation and freeze-drying, probably because of a slight degradation of acetates into glucosides. Traces of malvidin and peonidin 3-coumaroylglucosides were also observed. The pyruvic acid derivatives of malvidin- $(\mathrm{m} / \mathrm{z}, 707)$ and peonidin 3-coumaroylglucosides $(\mathrm{m} / \mathrm{z}, 677)$ could be tentatively identified on the basis of their molecular ions. Furthermore, their relative elution times are in good agreement with the order of elution of 66 anthocyanins analyzed by C18 reversed-phase HPLC (18). The presence of such derived anthocyanins in red wine has already been reported (18), but their presence in grape skin material is somewhat questionable. The presence of pyruvic acid, a yeast metabolite, formed by the microflora on the grape, could account for their formation. These derivatives were detected as minor constituents. Nevertheless, we cannot rule out the possibility that these derived structures may form during sample preparation (condensation of anthocyanins with pyruvic acid). Three other compounds $(m / z 657,653$, and 535) were detected. The first two compounds were probably the same as those present in fraction $\mathrm{C}$, as revealed by their similar MS/MS fragmentation patterns. The latter ion at $\mathrm{m} / \mathrm{z} 535$, when fragmented, gave rise to a positive ion at $\mathrm{m} / \mathrm{z} 331$, the same fragmentation pathway as that for malvidin 3-acetylglucoside. This observation was somewhat surprising and encouraged us to search for the existence (ion scans done in the different fractions) of peaks with masses corresponding to the other acylated anthocyanins. We could find two peaks corresponding not only to each acetylated but also coumaroylated anthocyanins. However, this pattern could not be observed for the glucosides, suggesting that the two species observed could correspond to isoforms where different $\mathrm{OH}$ groups of the sugar moiety could be esterified by acetic or coumaric acids or to the presence of different sugar stereoisomers.

Fractions $\mathrm{E}$ and $\mathrm{F}$ were both composed of anthocyanidinglucosides (compounds $\mathrm{q}$ to $\mathrm{u}$ ), and they were consequently pooled together for sensory studies. The $520 \mathrm{~nm}$ trace of this fraction showed the presence of the five anthocyanidinglucosides, malvidin and peonidin being the most abundant. A peak that eluted between petunidin and peonidin 3-glucoside could be identified as malvidin 3,5-diglucoside $(\mathrm{m} / \mathrm{z}, 655)$, an elution pattern in accordance with previously published data. Syringic acid $\left(\lambda_{\max } 277 \mathrm{~nm}\right.$ ), a catabolic derivative of the malvidin 3-glucoside, was tentatively identified on the basis of its retention time and spectral properties.

After fraction $\mathrm{F}$ was collected, the fractionation was halted and the material remaining in the stationary phase was colored 


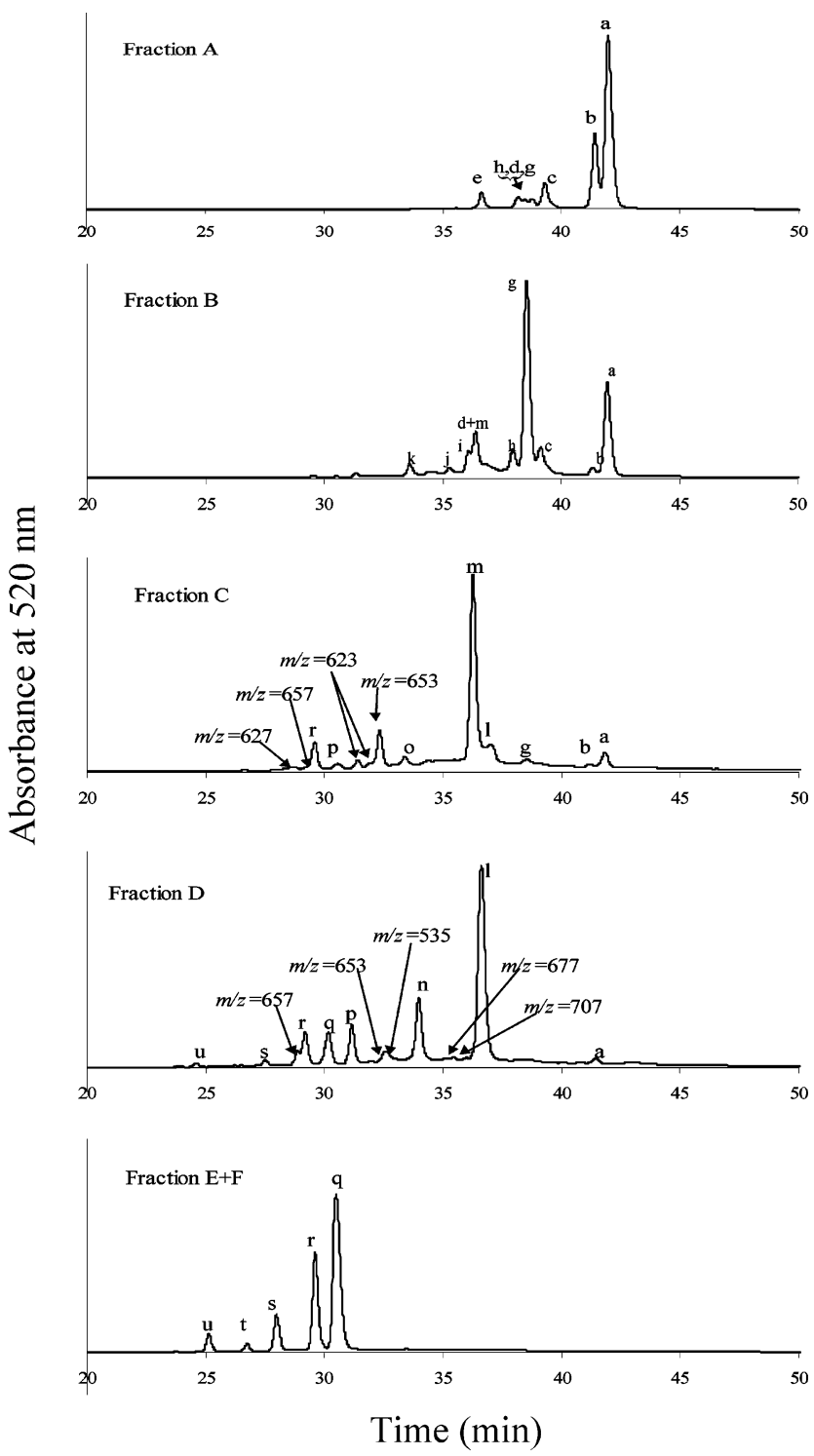

Figure 3. HPLC chromatograms at $520 \mathrm{~nm}$ of the six fractions collected. The peaks are labeled according to the codes given in Figure 1.

red and collected. On HPLC, this fraction was eluted as an unresolved hump at $520 \mathrm{~nm}$, which suggested that this fraction is polydisperse and devoid of monomeric anthocyanins. The ion scan method confirmed the absence of monomeric anthocyanins of any class. Furthermore, this fraction was resistant to thiolysis analysis performed under the standard conditions (2 min at $90{ }^{\circ} \mathrm{C}$ ). Structural characterization of this complex fraction requires further work.

\section{CONCLUSION}

Stepwise gradient elution coupled to MLCCC has been developed and allowed us to fractionate the different classes of grape anthocyanins with a single solvent system. This is the first time where a single solvent can be varied, without the use of appropritate tie-lines, in a multisolvent system, to enable a range of different phase polarities to further enhance separation of a complex matrix. These different fractions (classes of anthocyanin) can either be subjected to sensory studies (mouthfeel sensation or tactile sensation), color properties, or individually exploited for further fractionation to isolate individual pure components as reference compounds or starting materials in model reactions. A fraction devoid of monomeric anthocyanins and corresponding to the material left in the stationary phase is under structural characterization. This technique represents an alternative chromatographic tool to prepare not only anthocyanins but also other flavonoid fractions in sufficient amount to determine their intrinsic properties and to investigate the reactions in which they take part.

\section{ACKNOWLEDGMENT}

We wish to thank Professor Peter Høj and Drs. Elizabeth Waters and Mark Sefton from The Australian Wine Research Institute for their encouragement and critical reading of the manuscript. Dr. Markus Herderich is also thanked for his useful suggestions with the manuscript. We also thank Dr. Les Brown from AECS for fruitful discussion about the use of step gradient elution with MLCCC and Mr Holger Gockowiack for technical assistance. Société Française de Distillerie is thanked for providing polyphenolic extract from rosé wine pomace.

\section{LITERATURE CITED}

(1) Vidal, S.; Francis, L.; Williams, P.; Kwiatkowski, M.; Gawel, R.; Cheynier, V.; Waters, E. The mouth feel properties of polysacchrides and anthocyanins in a wine-like medium. Food Chem. 2004, in press.

(2) Brossaud, F.; Cheynier, V.; Noble, A. Bitterness and astringency of grape and wine polyphenols. Aust. J. Grape Wine Res. 2001, 7, 33-39.

(3) Cheynier, V.; Fulcrand, H.; Sarni, P.; Moutounet, M. Proceedings of Phenolics and Extraction conference, The Australian Society of Viticulture and Enology: Adelaide, Australia, 1997, and references therein.

(4) Böhm, H.; Boeing, H.; Hempel, J.; Raab, B.; Kroke, A. Flavonols, flavones and anthocyanins as natural antioxidants in food and their possible role in the prevention of chronic diseases. Z. Ernahrungswissenschaft 1998, 37, 147-163.

(5) Escribano-Bailon, T.; Alvarez-Garcia, M.; Rivas-Gonzalo, J. C.; Heredia, F. J.; Santos-Buelga, C. Color and stability of pigments derived from the acetaldehyde-mediated condensation between malvidin-3-O-glucoside and (+)-catechin. J. Agric. Food Chem. 2001, 49, 1213-1217.

(6) Atanasova, V.; Fulcrand, H.; Le Guerneve, C.; Cheynier, V.; Moutounet, M. Structure of a new dimeric acetaldehyde malvidin 3-glucoside condensation product. Tetrahedron Lett. 2002, 43, 6151-6153.

(7) Remy-Tanneau, S.; Guernevé, C. L.; Meudec, E.; Cheyneir, V. Characterization of a colorless anthocyanin-flavan-3-ol dimer containing both carbon-carbon and ether interflavanoid linkages by NMR and mass spectrometries. J. Agric. Food Chem. 2003, 51, 3592-3597.

(8) Degenhardt, A.; Knapp, H.; Winterhalter, P. Separation and purification of anthocyanins by High-Speed Countercurrent Chromatography and screening for antioxidant activity. J. Agric. Food Chem. 2000, 48, 338-343.

(9) Degenhardt, A.; Hofmann, S.; Knapp, H.; Winterhalter, P. Preparative isolation of anthocyanins by high-speed countercurrent chromatography and application of the color activity concept to red wine. J. Agric. Food Chem. 2000, 48, 5812-5818.

(10) Prieur, C.; Rigaud, J.; Cheynier, V.; Moutounet, M. Oligomeric and polymeric procyanidins from grape seeds. Phytochemistry 1994, 36, 781-784.

(11) Mateus, N.; Silva, A. M. S.; Vercauteren, J.; Freitas, V. d. Occurrence of anthocyanin-derived pigments in red wines. $J$. Agric. Food Chem. 2001, 49, 4836-4840.

(12) Souquet, J.-M.; Labarbe, B.; Le Guernevé, C.; Cheynier, V.; Moutounet, M. Phenolic composition of grape stems. J. Agric. Food Chem. 2000, 48, 1076-1080. 
(13) Souquet, J.-M.; Cheynier, V.; Brossaud, F.; Moutounet, M. Polymeric proanthocyanidins from grape skins. Phytochemistry 1996, 43, 509-512.

(14) Renault, J. H.; Thepenier, P.; Zeche-Hanrot, M.; Men-Olivier, L. L.; Durand, A.; Foucault, A.; Margraff, R. Preparative separation of anthocyanins by gradient elution centrifugal partition chromatography. J. Chromatogr., A 1997, 763, 345352.

(15) Ito, Y. Recent advances in counter-current chromatography. $J$. Chromatogr., A 1991, 538, 3-25.

(16) Rigaud, J.; Perez-Ilzarbe, X.; Ricardo-da-Silva, J. M.; Cheynier, V. Micro method for the identification of proanthocyanidin using thiolysis monitored by high-performance liquid chromatography. J. Chromatogr. 1991, 540, 401-405.

(17) Timberlake, C. F.; Bridle, P. Interactions between anthocyanins, phenolic compounds, and acetaldehyde and their significance in red wines. Am. J. Enol. Vitic. 1976, 27, 97-105.
(18) Heier, A.; Blaas, W.; Dross, A.; Wittowski, R. Anthocyanin analysis by HPLC/ESI-MS. Am. J. Enol. Vitic. 2002, 53, 7886.

Received for review August 11, 2003. Revised manuscript received December 8, 2003. Accepted December 14, 2003. This work was supported by Australia's grapegrowers and winemakers through their investment body the Grape and Wine Research and Development Corporation, with matching funds from the Australian federal government, and by the Commonwealth Cooperative Research Centre Program of Australia. The work was conducted by The Australian Wine Research Institute and Unité Mixte de Recherche Sciences pour l'Oenologie, INRA, and forms part of the research program of the Cooperative Research Centre for Viticulture in Australia.

JF034906A 